\title{
Speckle experiments in random lasers
}

\author{
Gijs van Soest, Frank J. Poelwijk, and Ad Lagendijk* \\ Van der Waals-Zeeman Instituut, Universiteit van Amsterdam, Valckenierstraat 65, 1018 XE, Amsterdam, The Netherlands
}

(Received 31 August 2001; published 18 March 2002)

\begin{abstract}
We present measurements of speckle in a random laser. We analyze its first-order statistics and show that, contrary to what might be expected for passive systems, analyses of the intensity distribution $P(I)$ and the speckle spot size do provide information about light transport inside the system. $P(I)$ is used to determine the degree to which an incident probe is amplified by the random laser. The shrinking speckle spot size reflects the change in path length distribution $P(\Lambda)$; we deduce that the average path length $\langle\Lambda\rangle$ in the studied random laser is two times longer above threshold than in a passive diffusive system.
\end{abstract}

DOI: 10.1103/PhysRevE.65.046603

\section{INTRODUCTION}

Random lasers are strongly scattering media that amplify light. There are striking similarities between these systems and more conventional lasers based on a gain medium enclosed in a cavity with two mirrors to provide optical feedback. An example is the observation of a threshold for lasing action and frequency narrowing in random lasers [1,2]. Evidently, the optical properties of random lasers are quite different from those of conventional lasers: the propagation of pump and fluorescence light is diffusive in a random laser. In contrast with cavity systems, scattering is actually advantageous. Since feedback is provided by multiple scattering, the random laser threshold is lowered by a stronger scattering, i.e., a shorter transport mean free path $l$, because the feedback is more efficient.

The random laser threshold has been a topic of particularly intense research in the past years. The ambiguity of the concepts of diffusive loss and feedback by multiple scattering, and the theoretical prediction of an intensity divergence, have spawned a continuing debate about what happens at, and above, the laser threshold. Recently, we demonstrated experimentally and theoretically [4] that this "explosion" does not occur, but rather is prevented by the dynamic interplay between laser light and excited population, resulting in gain saturation. The slow response of the population, compared to the light kinetics, starts a relaxation oscillation at the threshold crossing. We also established that the concept of loss in a conventional laser should be identified with the local magnitude of the intensity gradient in a random laser. The threshold condition now dictates that gain balances this transport locally. Consequently, a large gain coefficient $\kappa_{\mathrm{g}}(z)$ only builds up where the transport is large, i.e., near the interface of the medium, $z \leq 10 l$, where $z$ measures the distance from the interface. Like in a conventional laser, the gain grows linearly with pump rate below threshold, and is pump independent well above.

Speckle is the strongly fluctuating, grainy intensity pattern resulting from the interference of a randomly scattered coherent wave. It can be observed in space, time, and frequency. Some statistical characteristics of the speckle pattern contain information about the transport process [5-7]. We

\footnotetext{
*Corresponding author. Email address: adlag@phys.uva.nl
}

PACS number(s): 42.25.-p

discuss only speckle in space, i.e., a fluctuating intensity with angle.

If a coherent plane wave falls on a rough surface, a speckle pattern can be seen on a screen positioned at some distance from the scattering object. The scattered fields at a certain position on the screen comes from all points of the rough surface, and its random phases are distributed uniformly between 0 and $2 \pi$. The speckle is the sum of the electric field vectors of all the $N$ contributing partial waves. The summation constitutes a random walk in the complex plane, with a resulting field $E=1 / \sqrt{N} \Sigma_{k} a_{k} \exp \left(i \phi_{k}\right)$, where the summation is over the different contributions arising from different light paths [8].

The sum results in a Gaussian distribution for $E$, with most probable value $E=0$ and variance $\left\langle|E|^{2}\right\rangle$ $=\lim _{N \rightarrow \infty}(2 N)^{-1} \Sigma_{k}\left\langle\left|a_{k}\right|^{2}\right\rangle$. The observed intensity $I=|E|^{2}$ then follows a Rayleigh distribution

$$
P(I)=\frac{1}{\langle I\rangle} e^{-I /\langle I\rangle} .
$$

The typical speckle spot size depends on the characteristic distance along the screen on which the fields that contribute to the speckle dephase. The largest path length difference at a spot on the screen is caused by the partial waves arriving from opposite ends of the illuminated region of the scattering surface. Consequently, the typical angular size of a speckle spot is $\lambda / d$, if $d$ is the diameter of the illuminated region. This shows that a measurement of the speckle spot size, for instance, by the autocorrelation function of the speckle pattern, does not usually give information about light inside a scattering medium. The spot size in reflection depends mainly on the incident beam diameter. In transmission the most influential parameter is the sample thickness, which determines the degree to which an incident point source spreads in transport to the rear interface.

Light transport is affected by the presence of gain in a multiply scattering medium. The exponential dependence of the intensity on gain coefficient $\kappa_{\mathrm{g}}$ and path length $\Lambda$, given by $I=\exp \left(\kappa_{\mathrm{g}} \Lambda\right)$, enhances long paths more than short ones, resulting in a narrowed enhanced backscatter cone $[3,4]$. This modification of the path length distribution $P(\Lambda)$ is also expected to show in a measurement of speckle. By enhancing the weight of (far-reaching) long paths, the gain facilitates the spreading of light. 
The field of speckle experiments in random lasers is a largely uncharted territory. The only data available of the effect of gain on a speckle pattern produced by a probe beam are those of Wiersma and Lagendijk [9]. Gouedard et al. [10] and Noginov et al. [11] studied the related subject of coherence properties of the generated light. In this section we present experimental results concerning the intensity statistics and speckle spot size. In contrast with passive systems, these measurements do depend on parameters of light transport. There is no theory yet to compare the measurements with. We will give qualitative explanations of the results.

Theoretical studies of speckle in random lasers [12-15] invariably investigate speckle correlations. Although interesting, these correlations are experimentally not easily accessible, because of the stringent requirements they pose to the sample materials and the detection method. A further limitation of the theoretical efforts in this field is that they all rely on a stationary formalism, with fixed gain, so they encounter the explosion when approaching the laser threshold [4].

\section{EXPERIMENTAL DETAILS}

Spatial speckle can only be observed if the coherence length of the light is much larger than the maximum path length difference between partial waves contributing to speckle. We use a pulse from a frequency-doubled, $Q$-switched Nd:YAG (yttrium aluminum garnet) laser (Coherent Infinity $40-100$ ) as a probe pulse. Its duration is $2 \mathrm{~ns}$, and the linewidth is 1.5 times transform limited, from which a coherence length of $40 \mathrm{~cm}$ is deduced. This probe produces a speckle with a good visibility. The probe can be amplified by a dye with a large gain at $532 \mathrm{~nm}$, we choose coumarin 6 . In dye lasers this dye is usually dissolved in ethylene glycol, but unfortunately our $\mathrm{TiO}_{2}[16]$ colloid is not suspended well in this liquid. Hexylene glycol (2-methyl-2,4-pentanediol) is a good alternative. The fluorescence spectrum of coumarin 6 in hexylene glycol is nearly equal to that in ethylene glycol. Hexylene glycol also slows sedimentation of the scatterers by its high viscosity. We use an optical parametric oscillator (OPO; Coherent XPO) tuned to $482 \mathrm{~nm}$ as the pump source. The pump pulse duration is $1.7 \mathrm{~ns}$. The pump fluence is regulated by a set of polarizing Glan laser prisms, of which one can be rotated to vary the transmitted power.

The pump and probe pulses are incident simultaneously on the sample. The light in the medium then consists of amplified probe light, in which we are interested, and fluorescent "normal" random laser light. Because we want to do our experiments at large amplification, and the maxima of the gain curve $\kappa_{\mathrm{g}}(\lambda)$ and the fluorescence spectrum $L(\lambda)$ of a dye are usually close in wavelength, the probe and fluorescence cannot be fully separated spectrally. The fast decay of the dye also precludes a distinction in time to be made. We employ the coherence properties of the light to tell the probe and the fluorescence apart in the speckle measurement.

The suspension is contained in a round plastic container with dimensions of 6-mm depth $\times 10$-mm diameter, covered with a 4-mm-thick glass window. The sample is rotating slowly to prevent sedimentation and dye degradation. The dye concentration is $2 \mathrm{mM}$ and the transport mean free path

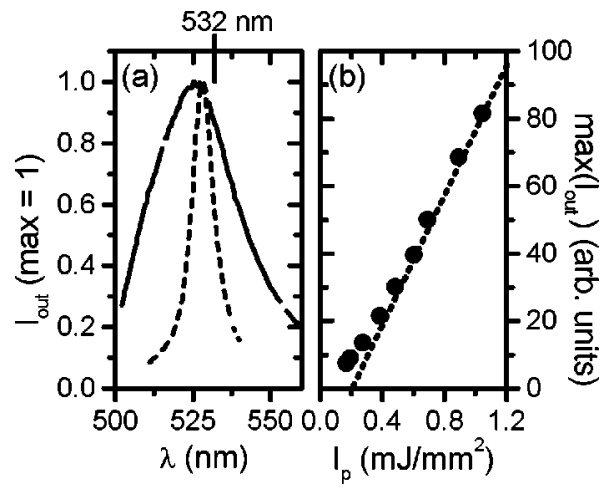

FIG. 1. (a) Line: normalized emission spectrum of a coumarin 6 solution in hexylene glycol, with $\mathrm{cw} 488-\mathrm{nm}$ excitation. Points: normalized emission spectra of the solution with $\mathrm{TiO}_{2}$ scatterers $(l=10 \mu \mathrm{m})$ below (solid line) and above threshold (dashed line). The pump source is a 482-nm pulse of duration $2.6 \mathrm{~ns}$. (b) Emitted intensity from the scattering solution at $527 \mathrm{~nm}$, near the maximum, as a function of pump intensity. The threshold is found to be at approximately $0.22 \pm 0.05 \mathrm{~mJ} / \mathrm{mm}^{2}$.

is $10 \mu \mathrm{m}$, from enhanced backscattering. This sample has a rather high threshold pump intensity of $I_{p}=0.22 \mathrm{~mJ} / \mathrm{mm}^{2}$, determined from the measurements shown in Fig. 1. We could not find a reliable, durable combination of dye and solvent that amplifies well at $532 \mathrm{~nm}$, and would suspend the colloid, with a lower threshold. The usual rhodamine-type dyes in random laser experiments amplify near $600 \mathrm{~nm}$. Since a sufficiently coherent pulsed probe source in this wavelength range was not available, we chose to use a different dye.

The speckle is recorded on a Kappa CF 8/1 FMC 8-bit charge-coupled device (CCD) camera. The light from the sample passes through an aperture, blocking stray light, a 532-nm interference filter with a transmission full width at half maximum of $1.0 \mathrm{~nm}$ to remove most of the fluorescence, and a neutral density filter. The image is a single shot exposure, because the sample is liquid so the speckle changes continuously. The probe beam diameter is $0.8 \mathrm{~mm}$. This produces a speckle that can be resolved well on the camera, with a large number of speckle spots. The pump spot is chosen to be larger $(2 \mathrm{~mm})$ to provide a large amplifying region for the probe light to propagate in. Figure 2 is a schematic of the

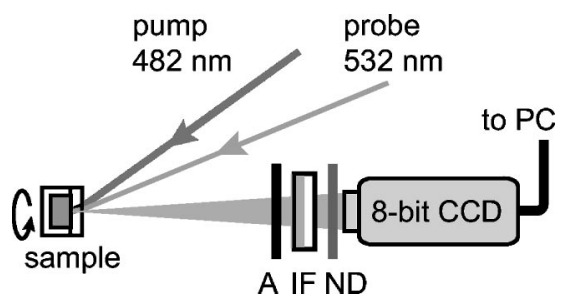

FIG. 2. Schematic of the setup for speckle experiments. The pump (diameter $2 \mathrm{~mm}$ ) and probe (diameter $0.8 \mathrm{~mm}$ ) reach the sample simultaneously. The sample is mounted on a motor, spinning it slowly to prevent sedimentation. The scattered and amplified probe light is collected on an 8 -bit $752 \times 582$ pixel CCD camera (Kappa CF 8/1 FMC), through an aperture (A), a 532-nm interference filter and one or more neutral density filters. The distance between sample and camera is $10 \mathrm{~cm}$. 

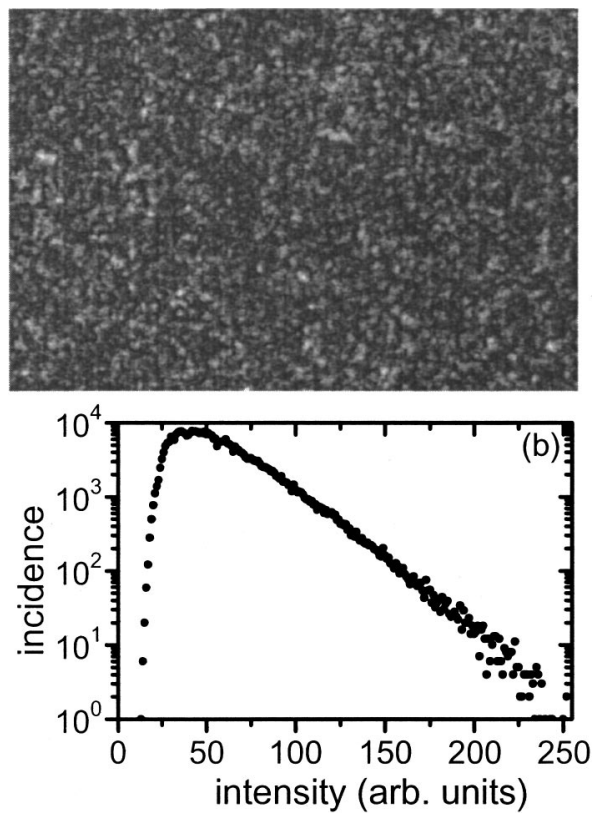

FIG. 3. (a) Speckle pattern on an 8-bit CCD camera of frequency-doubled Nd:YAG scattered and amplified by a $2 \mathrm{mM}$ Coumarin 6 solution in hexylene glycol with $\mathrm{TiO}_{2}$ scatterers $(l$ $=10 \mu \mathrm{m})$. The pump pulse from the OPO of wavelength $482 \mathrm{~nm}$ has an energy of $0.32 \mathrm{~mJ} / \mathrm{mm}^{2}$. (b) Intensity histogram of the image in (a). The Rayleigh distribution is offset by a background of incoherent fluorescence. The slope of the linear decrease is $1 /\langle I\rangle$.

experimental arrangement of sample and detection. It is important that the probe energy is small compared to the pump, and preferably also compared to the fluorescent emission from the system, to minimize the influence of the applied beam on the saturation. Under this condition the probe actually probes the system instead of partaking in the gain dynamics.

With this setup and sample, we study the first-order statistical properties of the speckle in the scattered, amplified probe light as a function of pump fluence. We investigate the intensity distribution and the average spot size.

\section{INTENSITY STATISTICS}

The Rayleigh distribution of speckle is a very robust phenomenon. The only requirements are uncorrelated phases of the scattered light and the independence of the amplitude $a_{k}$ and phase $\phi_{k}$. It does not make a principal difference whether the scattered light has actually traveled inside the scattering medium or is just reflected off the surface. Considered this way, a measurement of speckle intensity statistics does not promise to be an effective method to obtain new information about random lasers. It does, however, provide access to a measurement that is otherwise difficult to perform: the degree to which the incident probe is amplified by the system.

Figure 3(a) shows an image of the speckle pattern in scattered and amplified probe light from a coumarin $6 / \mathrm{TiO}_{2}$ random laser. The drawback of the large pump spot is a larger fluorescence component in the image.

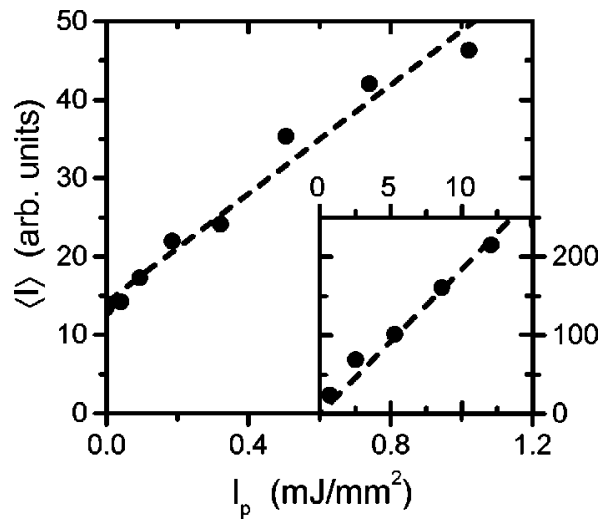

FIG. 4. Average intensity derived from Rayleigh statistics as a function of pump fluence, for a fixed probe intensity of $59 \mu \mathrm{J} / \mathrm{mm}^{2}$. The amplified intensity grows linearly with pump fluence. For comparison, we plot the mean intensity as a function of the probe fluence (in units of $0.01 \mathrm{~mJ} / \mathrm{mm}^{2}$ ) in the inset. The pump intensity is high: $2.9 \mathrm{~mJ} / \mathrm{mm}^{2}$. The lines are linear fits to the data.

The intensity histogram of the speckle in Fig. 3(a) is shown in Fig. 3(b). The histogram has an unusual feature compared to Eq. (1): it only starts to show Rayleigh statistics above intensity 50. The lower intensities are incoherent fluorescence, giving each pixel an offset. The average intensity can be determined from the slope of the exponential decrease for higher intensities. A plot of the fluorescent intensity (the offset) as a function of pump fluence reproduces Fig. 1(b), providing evidence that the saturation dynamics of the system is not significantly influenced by the amplified probe pulse.

We extract the average intensity of the amplified probe from the slope of the intensity statistics plotted in the manner of Fig. 3. The dependence of the average intensity of the speckle or the intensity of the amplified probe on pump fluence is plotted in Fig. 4. The exposures taken at the highest pump intensities are overexposed, but if the average intensity can still reliably be obtained from the low intensity part of the negative exponential, it is included in the graph. A measurement in which the intensity on the camera is attenuated using a neutral density filter shows that the linear behavior of Fig. 4 persists up to the highest pump intensity of $2.9 \mathrm{~mJ} / \mathrm{mm}^{2}$, amounting to an amplification factor $\approx 10$. The linearity with probe fluence (inset of Fig. 4) shows that the amplification factor is independent of probe fluence, providing more evidence that the probe does not affect the gain in the system.

\section{SPECKLE SPOT SIZE}

As discussed earlier, a measurement of the speckle spot size yields the transverse dimension of the coherent source on the scattering surface. In a random laser this may very well depend on the pump energy, since a larger gain allows the light to spread further, enhancing the contribution of long paths. A relation between the transverse dimension of the amplifying volume and the threshold has been demonstrated [2].

The spot size is measured by calculating the two- 


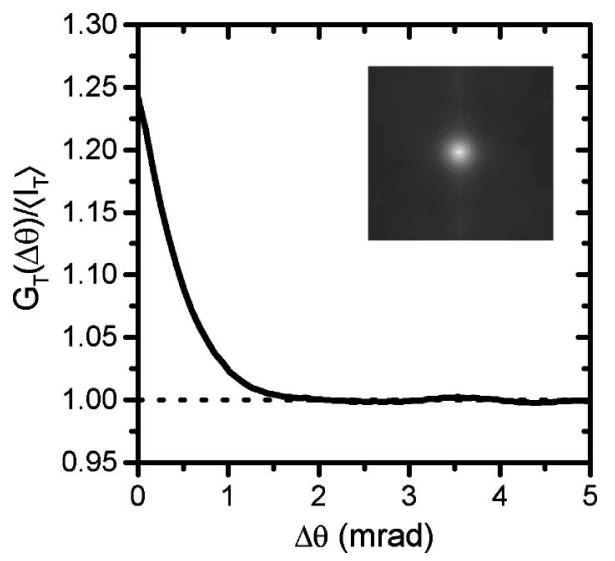

FIG. 5. A cross section through the autocorrelate $G_{T}(\Delta \theta)$ of the data in Fig. 3, normalized to the average total intensity $\left\langle I_{T}\right\rangle$. The contrast is reduced from 2 to 1.25 due to the incoherent background, complicating the determination of the first zero $\theta_{0}$. The two-dimensional autocorrelate itself is shown in the inset.

dimensional intensity autocorrelate $G_{I}(\Delta \theta)=\langle I(\theta) I(\theta$ $+\Delta \theta)\rangle$ of a speckle pattern as in Fig. 3(a). For a circular illumination spot of diameter $d$ by purely coherent light the autocorrelation is

$$
G_{I}(\Delta \theta)=\langle I\rangle^{2}\left[1+A\left(\frac{d}{\lambda} \Delta \theta\right)\right],
$$

where $\Delta \theta$ is the angular distance between two points on the screen, and $A(u)=\left[2 J_{1}(u) / u\right]^{2}$ with $J_{1}$ the first-order Bessel function. $A(u)$ has the same functional dependence as the Airy diffraction pattern of a circular aperture, and so the first zero is expected at $\Delta \theta=\theta_{0}=1.22 \lambda / d$, providing a measure for the speckle spot size. The contrast between the maximum at zero and the value at large $\Delta \theta$ is a factor of 2: $G_{I}(\Delta \theta \gg \lambda / d)=\langle I\rangle^{2}$ and $G_{I}(0)=2\langle I\rangle^{2}$.

Our diffuse light source is, however, partly incoherent due to the contribution of fluorescence. The consequences for the intensity autocorrelate are shown in Fig. 5, showing a cross section through the autocorrelate of the data in Fig. 3(a). The speckle contrast is reduced to $1+\left(\langle I\rangle /\left\langle I_{T}\right\rangle\right)^{2}$ [17], where $\langle I\rangle$ is the average intensity of the amplified probe and $\left\langle I_{T}\right\rangle$ $=\langle I\rangle+I_{F}$ is the average total intensity, including the fluorescence intensity $I_{F}$. For the data in Fig. $3,\langle I\rangle \approx I_{F}$ and so the contrast in the autocorrelate is reduced to a factor of 1.25 . It becomes difficult to determine the position of the first zero in the autocorrelate due to this lower contrast.

Another change with respect to the fully coherent situation is the disappearance of the flat top for $G_{I}(\Delta \theta=0)$, predicted by Eq. (2). We have no explanation for this sharpening. The resulting $G_{T}(\Delta \theta)\left[=G_{I_{T}}(\Delta \theta)\right]$ is normalized to $\left\langle I_{T}\right\rangle$ and analyzed quantitatively by modeling it with a function

$$
G_{m}(\Delta \theta)=1+\left[\frac{\langle I\rangle}{\left\langle I_{T}\right\rangle}\right]^{2} e^{-\Delta \theta / \theta_{c}}
$$

There is no reason for comparing $G_{T}$ with precisely this $G_{m}$ but like appearances. The fit with $G_{m}$ provides a reproduc-

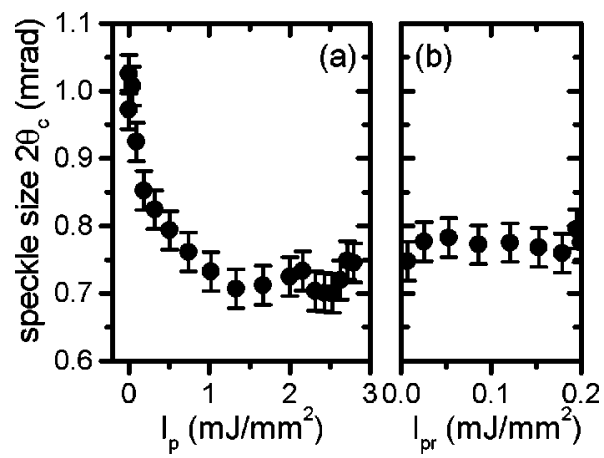

FIG. 6. Characteristic decay angle of the autocorrelate, as measured by a fitting $G_{T}$ with $G_{m}$, given in Eq. (3). (a) For large pump fluence the speckle spots get significantly smaller compared to the case without gain, signifying that the amplification assists the spatial spreading of the probe light. The probe fluence is $59 \mu \mathrm{J} / \mathrm{mm}^{2}$. (b) With varying probe energy at a pump fluence of $2.9 \mathrm{~mJ} / \mathrm{mm}^{2}$, the speckle size is constant, demonstrating the role of the amplification in the effect in (a).

ible way to quantify the speckle spot size. $\langle I\rangle$ and $\left\langle I_{T}\right\rangle$ are determined directly from the data by taking the slope of the intensity histogram and the total average, respectively, so $\theta_{c}$ is the only free parameter in a fit with $G_{m}$. The characteristic angle $\theta_{c}$ is smaller than $\theta_{0}$ by a constant factor $\lesssim 1$.

The $\theta_{c}$ are plotted as a function of pump fluence in Fig. 6(a). The speckle spots are found to shrink as the pump fluence is increased from 0 to $\approx 1 \mathrm{~mJ} / \mathrm{mm}^{2}$, after which their size is approximately constant. The optical resolution of the imaging system is $\approx 0.1 \mathrm{mrad}$. Apparently the source of diffuse light producing the speckle becomes larger if the pump fluence is larger. This is consistent with the notion that mainly the long paths are amplified in a random laser. If only the intensity is increased, without actually changing the amount of amplification, the speckle size is constant as shown in Fig. 6(b).

Without the pump, the speckle size is set by the probe beam diameter of $0.8 \mathrm{~mm}(80 l)$, and for the highest pump energies the equivalent source size increases to approximately 1.5 times this value. For high pump fluence the speckle does not get smaller. This is consistent with the result [4] that far above threshold the local equilibrium gain is clamped at the local loss level, so the gain coefficient $\kappa_{\mathrm{g}}$ at position $\mathbf{r}$ does not depend on the pump fluence.

\section{DISCUSSION}

The measurement of intensity statistics reveals the amplification factor for an external laser seed in the slope of the speckle intensity histogram in Fig. 3(b). The results in Fig. 4 show that a random laser works as a linear amplifier for a probe beam over the entire range of pump rates.

The laser threshold does not appear in a measurement of the average probe intensity $\langle I\rangle$ as a function of pump fluence $I_{\mathrm{p}}$. Above threshold the probe light behaves as a coherent contribution to the "normal" (amplified fluorescence) random laser light, and hence the dependence on pump rate is linear, the gain saturation being effected by the normal laser 
light. The threshold arises, however, because spontaneous emission dominates. Part of the fluorescence is not amplified [18] and does not contribute to the laser intensity as plotted in Fig. 1(b). The probe does not suffer this loss and can profit fully from the gain that increases linearly with pump rate below threshold.

The longer average path length in a random laser directly influences the speckle spot size. Long paths spread further, and if these get a larger relative weight in the diffuse average, the coherent source has a larger apparent diameter. We demonstrate this effect in Fig. 6: the speckle spot size is found to diminish, with increasing pump rate, up to the threshold region. Far above threshold the speckle size is constant, reflecting the effect of saturation. The change in speckle spot size is similar in appearance and origin to the narrowing of the EBS cone, which also shows the effect of saturation in the same way [4].

The constant speckle spot size as a function of probe fluence shows that this shrinkage is indeed an effect of the gain: although the larger scattered intensity produced by a higher incident probe fluence visually seems to produce a larger spot, the intensity distribution is independent of the amount of probe light. Amplification truly changes the distribution of path lengths, and so the distribution of amplified probe intensity on the surface.

A quantitative assessment of the modification of the path length distribution $P(\Lambda)$ is not possible with the available theory. The theory of Ref. [4] takes into account only one spatial variable, whereas the transverse dimensions are clearly needed for describing the lateral spreading of the probe. Even in one dimension $P(\Lambda)$ cannot be determined in the absence of a stationary form for the diffusing density. Recalling that the rms traveled distance in a random walk of length $\Lambda$ is $\sqrt{l \Lambda / 3}$, we conclude that the average path length $\langle\Lambda\rangle$ becomes $(1.5)^{2}=2.25$ times larger in this sample.

We conclude with a proposal for a supplementary experiment. In a solid random laser, with scatterers at fixed positions, one can correlate subsequent exposures. In this way it is possible to measure the correlation $C\left(\Delta I_{\mathrm{p}}\right)=\left\langle I\left(I_{\mathrm{p}}\right) I\left(I_{\mathrm{p}}\right.\right.$
$\left.\left.+\Delta I_{\mathrm{p}}\right)\right\rangle$. It is derived from the classic $C_{1}$ correlation [6], measuring the gradual change in a speckle pattern with a variation of the incident field (usually, e.g., angle or frequency). $C_{1}\left(\Delta I_{\mathrm{p}}\right)$ will reflect the change in spot size presented above, but then in a measurement that is theoretically better controlled: the autocorrelate that we use mixes different output channels by adding different angles.

The traditional $C_{1}\left(\Delta k_{\perp}\right)$ has a weak dependence on the absorption, appreciable if $1 / L_{\mathrm{a}}^{2}$ is not negligible compared to $k_{\perp}^{2}$, so for small angles [7]. Upon replacing $L_{\mathrm{a}}$ by $-L_{\mathrm{g}}$, which is allowed for a weak probe that does not saturate the gain, we gauge that $C_{1}$ should be appreciably larger than the passive case for rotation angles smaller than $\left(k L_{\mathrm{g}}\right)^{-1}$. This estimate applies to the transmission of a sample with (essentially) homogeneous gain. The analysis of Burkov and Zyuzin [12] suggests the same relative change due to gain for short- and long-range correlations.

\section{CONCLUSIONS}

The experiments presented in this paper show that the modification of the transport properties by gain in a random laser influences the first-order statistics of the speckle observed in scattered and amplified probe light. These speckle statistics are easily accessible to experiment, and provide insight into the gain dynamics and light transport in random lasers. The intensity histogram provides a measurement of the amplification factor for an external seed, because the fluorescent emission does not show Rayleigh statistics and can therefore be separated from the probe. The decrease of the speckle spot size shows that the apparent size of the diffuse source grows with gain, by enhancement of long light paths.

\section{ACKNOWLEDGMENTS}

This work is part of the research program of the "Stichting voor Fundamenteel Onderzoek der Materie (FOM)," which is financially supported by the "Nederlandse Organisatie voor Wetenschappelijk Onderzoek (NWO)."
[1] N.M. Lawandy, R.M. Balachandran, A.S.L. Gomes, and E. Sauvain, Nature (London) 368, 436 (1994).

[2] G. van Soest, M. Tomita, and A. Lagendijk, Opt. Lett. 24, 306 (1999).

[3] D.S. Wiersma, M.P. van Albada, and A. Lagendijk, Phys. Rev. Lett. 75, 1739 (1995).

[4] G. van Soest, F.J. Poelwijk, R. Sprik, and A. Lagendijk, Phys. Rev. Lett. 86, 1522 (2001).

[5] A.Z. Genack, in Scattering and Localization of Classical Waves in Random Media, edited by P. Sheng (World Scientific, Singapore, 1990).

[6] S. Feng, C. Kane, P.A. Lee, and A.D. Stone, Phys. Rev. Lett. 61, 834 (1988).

[7] J.F. de Boer, Ph.D. thesis, Universiteit van Amsterdam, 1995.

[8] J.W. Goodman, in Laser Speckle and Related Phenomena, 2nd ed., edited by J.C. Dainty (Springer, Berlin, 1984).
[9] D.S. Wiersma and A. Lagendijk, Physica A 241, 82 (1997).

[10] C. Gouedard, D. Husson, C. Sauteret, F. Auzel, and A. Migus, J. Opt. Soc. Am. B 10, 2358 (1993).

[11] M.A. Noginov, S.U. Egarievwe, N. Noginova, H.J. Caulfield, and J.C. Wang, Opt. Mater. 12, 127 (1999).

[12] A.A. Burkov and A.Yu. Zyuzin, Phys. Rev. B 55, 5736 (1997).

[13] A.A. Burkov and A.Yu. Zyuzin, Pis'ma Zh. Eksp. Teor. Fiz. 63, 841 (1996) [JETP Lett. 63, 878 (1996)].

[14] V. Freilikher, M. Pustilnik, and I. Yurkevich, Phys. Rev. B 56, 5974 (1997).

[15] A.Yu. Zyuzin, Phys. Rev. E 51, 5274 (1995).

[16] Sachtleben Chemie GmbH, Hombitan RCL 66 Titandioxid, $89+\% \mathrm{TiO}_{2}$ rutile, surface coated with alumina/silica, average particle size $220 \mathrm{~nm}$.

[17] G. Parry, in Laser Speckle and Related Phenomena, 2nd ed., edited by J.C. Dainty (Springer, Berlin, 1984).

[18] G. van Soest and A. Lagendijk, Phys. Rev. E (to be published). 